\title{
Erratum: Magnetic structure and magnetization of helical antiferromagnets in high magnetic fields perpendicular to the helix axis at zero temperature [Phys. Rev. B 96, 104405 (2017)]
}

\author{
David C. Johnston \\ Ames Laboratory and Department of Physics and Astronomy, Iowa State University, Ames, Iowa 50011, USA
}

(Received 16 September 2018; published 28 September 2018)

DOI: 10.1103/PhysRevB.98.099903

The prefactors of 2 in the second and third terms of Eqs. (6) and (9a) are typographical errors and should not be present. In addition, a typographical error in the normalization of the energies is present: energies are normalized by $S^{2} J_{2}$ instead of $S J_{2}$. Hence the reduced magnetic field in Eq. (7) is $h_{x}=g \mu_{\mathrm{B}} H_{x} /\left(S J_{2}\right)$ and similarly for the reduced critical field $h_{\mathrm{c}}$ and transition field $h_{\mathrm{t}}$. The correct Eqs. (6) and (9) are

$$
\begin{aligned}
E_{n} & =\frac{S}{2}\left\{S J_{0}+S J_{1}\left[\cos \left(\phi_{n+1}-\phi_{n}\right)+\cos \left(\phi_{n-1}-\phi_{n}\right)\right]+S J_{2}\left[\cos \left(\phi_{n+2}-\phi_{n}\right)+\cos \left(\phi_{n-2}-\phi_{n}\right)\right]\right\}-\mu_{n} H_{x} \cos \phi_{n}, \\
\frac{E_{n}}{S^{2} J_{2}} & =\frac{1}{2}\left\{J_{02}+J_{12}\left[\cos \left(\phi_{n+1}-\phi_{n}\right)+\cos \left(\phi_{n-1}-\phi_{n}\right)\right]+\left[\cos \left(\phi_{n+2}-\phi_{n}\right)+\cos \left(\phi_{n-2}-\phi_{n}\right)\right]\right\}-h_{x} \cos \phi_{n}, \\
\frac{E_{\text {ave }}}{S^{2} J_{2}} & =\frac{1}{n_{\lambda}} \sum_{n=0}^{n_{\lambda}-1} \frac{E_{n}}{S^{2} J_{2}} .
\end{aligned}
$$

The energy normalization typographical error is also present in the left sides of Eqs. (13a), (13c), and (19) and in the expression for the reduced field $h_{x}$ in the abscissa labels in Figs. 12 to 30. All numerical values presented in the tables and figures are obtained from the correct equations and are hence unchanged. Two lines below Eq. (14), " $k d$ " should be " $k d / \pi$ ". 